This item was submitted to Loughborough's Research Repository by the author.

Items in Figshare are protected by copyright, with all rights reserved, unless otherwise indicated.

\title{
Experimental and numerical methods to analyse deformation and damage in random fibrous networks
}

PLEASE CITE THE PUBLISHED VERSION

https://doi.org/10.1007/978-3-030-34851-9_9

PUBLISHER

Springer International Publishing

VERSION

AM (Accepted Manuscript)

PUBLISHER STATEMENT

The final authenticated version is available online at https://doi.org/10.1007/978-3-030-34851-9.

LICENCE

CC BY-NC-ND 4.0

\section{REPOSITORY RECORD}

Sozumert, Emrah, Emrah Demirci, and Vadim Silberschmidt. 2019. "Experimental and Numerical Methods to Analyse Deformation and Damage in Random Fibrous Networks". figshare.

https://hdl.handle.net/2134/9790898.v1. 


\title{
Chapter 8
}

\section{Experimental and Numerical Methods to Analyse Deformation and Damage in Random Fibrous Networks}

\author{
Emrah Sozumert, Emrah Demirci, and Vadim V. Silberschmidt
}

\begin{abstract}
Deformation and damage behaviours of random fibrous networks are investigated with experimental and numerical methods at local (fibre) and global (specimen) levels. Nonwoven material was used as an example of fibrous network, with its individual fibres were extracted and tested with a universal testing system in order to assess their material properties. The fibres demonstrated a nonlinear time-dependent response to stretching. For analysis of notch sensitivity, undamaged nonwoven specimens and those with various notch shapes were analysed with fabriclevel tensile tests and finite-element simulations. A level of strains around notch tips was tracked in simulations, demonstrating that the material was notch-sensitive, but load-transfer mechanisms were different than those in standard homogeneous materials. The notch shape also affected the rate of damage growth in the main directions. A good agreement between experimental and numerical damage patterns was observed. Also, the notch shape affected strength and toughness of the fibrous network.
\end{abstract}

Key words: Fibrous networks · Deformation · Damage $\cdot$ Finite-element modelling $\cdot$ Nonwovens

\subsection{Introduction}

This research focuses on mechanics of deformation and damage of random fibrous networks that are ubiquitous in natural environments (e.g. collagens) and synthetic materials, such as nonwovens. They can be produced by living microorganisms (such as bacteria) in aqueous-environment (Gao et al., 2016a). They form a membrane in the

Emrah Sozumert · Emrah Demirci · Vadim V. Silberschmidt

Loughborough University, UK,

e-mail: e.sozumert@lboro.ac.uk, e.demirci@lboro.ac.uk,

v.silberschmidt@lboro.ac.uk 
form of amniotic sac to provide a protective environment for a developing embryo in human body (Buerzle and Mazza, 2013). Random fibrous networks can be produced with an electrospinning process and merging fibrous networks are used to host cells in a process of repairing skin injuries (Norouzi et al., 2015). Therefore, there is a growing interest in the improvement of existing fibrous networks and developing new ones thanks to their broad range of potential applications. This inevitably attracts the attention of researchers to the deformation and damage mechanisms of such networks. This paper presents a full methodology for mechanical characterisation of deformation and damage of a random fibrous network based on a combination of experimental and numerical approaches (from single-fibre to fabric tests).

\subsubsection{Background}

Random fibrous networks are discontinuous materials with their complex microstructures. Considering their deformation and damage behaviour, such networks have advantageous compared to traditional continuous materials, such as metals. For instance, Gao et al. (2016b) and Ridruejo et al. (2015) reported that a notch in a random fibrous network can improve material stiffness. Researchers in academia and industry investigate microstructures of these materials since microstructural parameters, such as alignments of fibres, influences their effective mechanical properties, such as the strength of the network, at macro-level (Sabuncuoglu et al., 2012). Additionally, these parameters can be tuned to enhance mechanical properties (Ovaska et al., 2017).

To study these materials, a nonwoven fibrous network with random orientation distribution of fibres is considered as an example material in this research. Fibres of this material underwent a thermal-bonding process and were bonded together by high pressure and temperature. Conditions of the web-bonding process, such as its temperature, controls mechanical properties of this type of random fibrous networks (Michielsen et al., 2006). In general, the manufacturing process results in anisotropy, with three main orthogonal directions: machine direction (MD), cross direction (CD), and thickness direction (TD). Fibres are preferentially aligned along MD as a result of manufactures, and, as tensile load applied, an anisotropic mechanical response is observed, with higher stiffness in MD than that in CD (Demirci et al., 2012).

The aim of this study is to present experimental and numerical methods used to investigate deformation and damage mechanisms of fibrous networks. In experimental methods, readers can find the techniques for characterisation of tensile behaviour of individual fibres as well as random fibrous networks. Fibres extracted from nonwoven networks were tested in a micro tester to assess their time-dependent elastoplastic properties; while fabric tests were performed to observe deformation and damage evolutions at macro-scale. The data obtained from the single-fibre tests were used as input to numerical investigations (finite-element(FE) simulations). Scanning Electron Microscopy (SEM) and Micro Computed Tomography (micro CT) were employed to analyse microstructure of nonwoven samples and to provide data for

Page: 132 job:ABK_editor macro: svmult.cls date/time: 4-Sep-2019/22:46 
development of parametric FE models. Microstructural features were directly incorporated into these models for samples without notches and with various notches, mimicking the microstructure of a selected random fibrous network. In order to evaluate the evolution of local deformation and fracture behaviour in fibrous networks, the FE models were stretched along their main direction - MD. Global and local (at fibre level) responses of the fibrous networks were recorded with strain distributions over central area and in the vicinity of notch tips tracked.

\subsubsection{Fundamental Concepts}

Complexity of deformation and damage mechanisms of random fibrous networks stems from their complex microstructure described in terms of orientation distribution of fibres, nonlinear behaviour of individual fibres and fibre curvature. The main source of nonlinearity related to orientation distribution of fibres is randomness. Two types of randomness can be encountered in a fibrous network:

(i) randomness in spatial alignment of fibres and

(ii) randomness in their orientation.

Such microstructure of fibrous networks dictates introduction of randomly distributed fibres in network domain. A fibre can be defined with a random orientation angle $\phi_{1}$, and its centre in $x-y$ coordinate system $C_{1}$ (Fig. 8.1 (a)). The centre of this fibre can be placed in a different location $C_{2}$ (Fig. 8.1 (b)), to model the randomness in spatial alignments of fibres, or in the same location $C_{1}$ with a different orientation angle $\phi_{3}$ (Fig. 8.1 (c)) (randomness in orientation of fibres).

Although the adjective random is used for fibrous networks, alignment of fibres in them are usually not fully random. In cases when the alignments of fibres are random, these random fibrous networks are called fully-random fibrous networks.

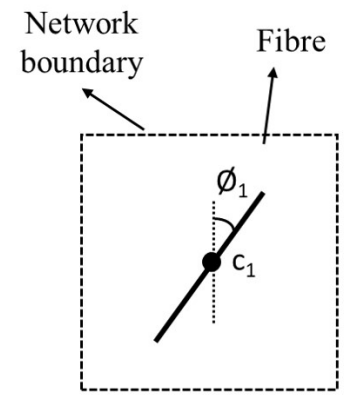

(a)

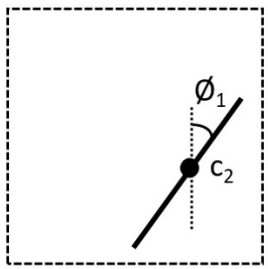

(b)

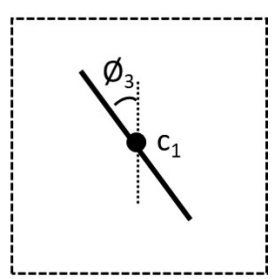

(c)

Fig. 8.1 Introduction of fibre with three different configurations: (a) reference configuration, (b) same orientation and different centre, (c) different orientation and same centre 
Furthermore, orientation distribution of fibres is an important element, affecting mechanical properties of fibrous networks. In addition to random spatial distribution, some of these networks have preferential orientations of fibres, with some principal directions. It was reported that different stiffness and toughness measurements were obtained in the networks with a non-uniform orientation distribution of fibres (Yang et al., 2015). To express the randomness in orientation distribution of fibres, Cox (1952) introduced a distribution function for fibrous mats. This is also known as orientation distribution function (ODF). Determination of this function is important for elucidating actual microstructure of fibrous networks, as it is a fundamental input into numerical models. In nonwoven materials, there are three main directions defining orientation of fibres in corresponding material: MD (machine direction), $\mathrm{CD}$ (cross direction), TD (thickness direction). In fact, the machine direction is the direction coinciding with that of the production line and perpendicular to the cross direction. This orientation distribution of fibres can be assessed from SEM images by employing Fourier- or Hough-transform-based methods (Demirci et al., 2012; Ghassemieh et al., 2002; Kim and Pourdeyhimi, 2001). They are based on detection of fibres in fibrous networks. A sample SEM image and its computed ODF are presented in Fig. 8.2.

The third nonlinearity source is fibre curvature (in other words, fibre crimp or curliness), e.g. studied by Hearle and Stevenson (1963). A fibre with crimp shows negligible stiffness until it becomes fully straight. Overall mechanical behaviour of a fibrous network is directly affected by crimp of fibres (Kumar and Rawal, 2017; Shiffler, 1995). Crimp geometry was described by a sinusoidal-like wave (Rawal, 2006). Additionally, crimp can increase material ductility and toughness (Sozumert et al., 2018). Another source of nonlinearity in complex mechanical behaviour of random fibrous networks is a nonlinear material response of individual fibres (Farukh et al., 2012; Sabuncuoglu et al., 2013). Polymer fibres with diameters at micro-level were tested under tensile testing conditions in various investigations; their timedependent material response was also obtained (Sabuncuoglu et al., 2013). Clearly, time-dependent material properties of fibres alter a tensile response of random fibrous

(a)

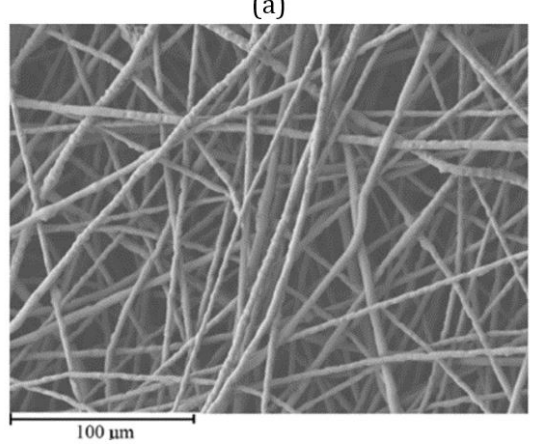

(b)

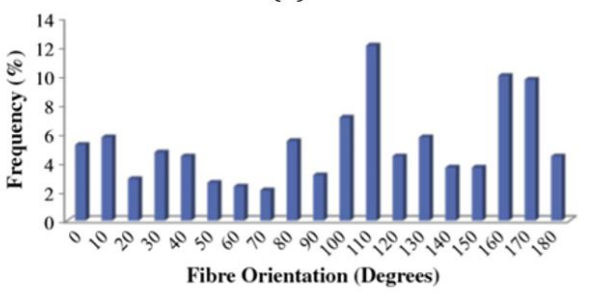

Fig. 8.2 SEM image of nonwoven (a) and its computed ODF (b) (Demirci et al., 2012) 
networks. Testing of individual fibres is, however, not always feasible (particularly for fibres with diameters at nano-scale); especially when fibres can not be easily removed from a fibrous network for testing. Hence, alternative ways, such as methods based on Atomic Force Microscopy (AFM) (Cheng and Wang, 2008; Tan et al., 2005) and/ or numerical-based methods (Gao et al., 2017), can be used for characterisation of individual fibres. From the microscopic point of view, deformation and damage in random fibrous networks can be explained with few steps at micro-level (Farukh et al., 2014b; Ridruejo et al., 2011):

(i) debonding of fibre under low level of strain;

(ii) re-orientation of fibres towards loading direction;

(iii) straightening of fibres, and

(iv) stretching and

(v) failure of individual fibres.

Microscopic appearance of a nonwoven felt subjected to axial stretch at maximum load is shown in Fig. 8.3 (a) and, as the load increased, damage occurred due to failure of fibre bonds followed by failure of fibres (Fig. 8.3 (b)) Ridruejo et al. (2010). This shows damage starts at local level and spreads to global level. Since understanding of local damage and deformation micro-mechanisms of random fibrous networks is important for this research, inserting an artificial crack (i.e. notch) can ensure the localisation of damage around certain areas for a better and easier analysis. Therefore, the effect of local damage on structural integrity and material behaviour can be evaluated with notch sensitivity.

Finite-element (FE) method is used to simulate deformation and fracture of fibrous networks and to reveal information about fundamental micromechanisms underpinning these phenomena, such as in (Ghassemieh et al., 2002; Isaksson and Hägglund, 2009; Koh et al., 2013; Kulachenko and Uesaka, 2012; Mueller and Kochmann,

(a)

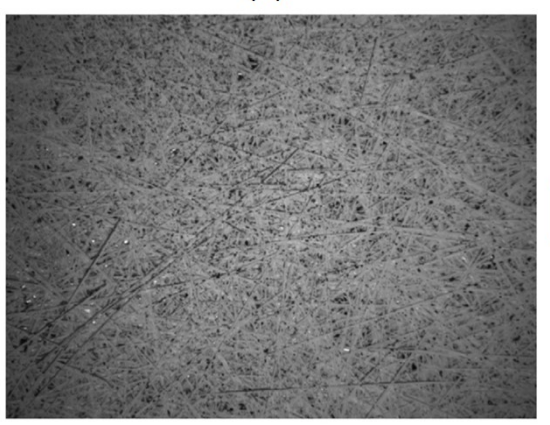

Nonwoven at maximum load (b)

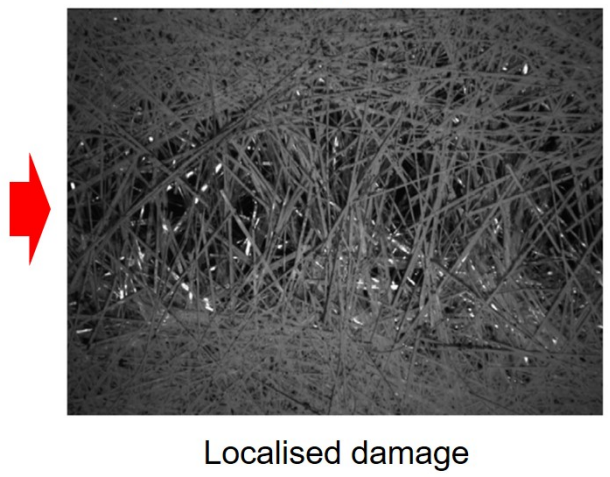

Fig. 8.3 Optical micrographs of nonwoven felt under tensile deformation at maximum load (a) and localised damage as stretched further (b) (modified from Ridruejo et al., 2010) 
2004). Notch-sensitivity in random fibrous networks composed of brittle fibres is investigated in Isaksson and Hägglund (2009); Koh et al. (2013); Ridruejo et al. (2010) and, as for ductile fibres, in Ridruejo et al. (2010). The fibrous networks with ductile fibres, such as some nonwovens, demonstrate a blunted notch area reducing stress-concentration. The fibrous networks with brittle fibres, such as gelatine electrospun scaffolds, however, protected the network morphology around the notch tip.

\subsection{Experimentation}

\subsubsection{Material}

A thermally bonded calendared $30 \mathrm{~g} / \mathrm{m}^{2}$ nonwoven composed of monocomponent polypropylene fibres is used as an example random fibrous network. To enhance mechanical properties by tuning crystallinity, after the propylene fibres were extruded, they were stretched (Jubera et al., 2014). This process was followed by lying down the spun fibres randomly on a moving conveyed belt. The fibres on the belt showed non-uniformity in their orientation distribution. At the subsequent stage, i.e. calendaring process, heat and pressure were simultaneously applied to bond fibres. This manufacturing process caused a clear distinction in bonded and unbonded regions of the network. The bonded fibres as bond points with a spatial pattern and unbonded bridging fibres as fibre matrix are shown in Fig. 8.4.

Fig. 8.4 SEM image of $30 \mathrm{~g} / \mathrm{m}^{2}$ point-bonded nonwoven

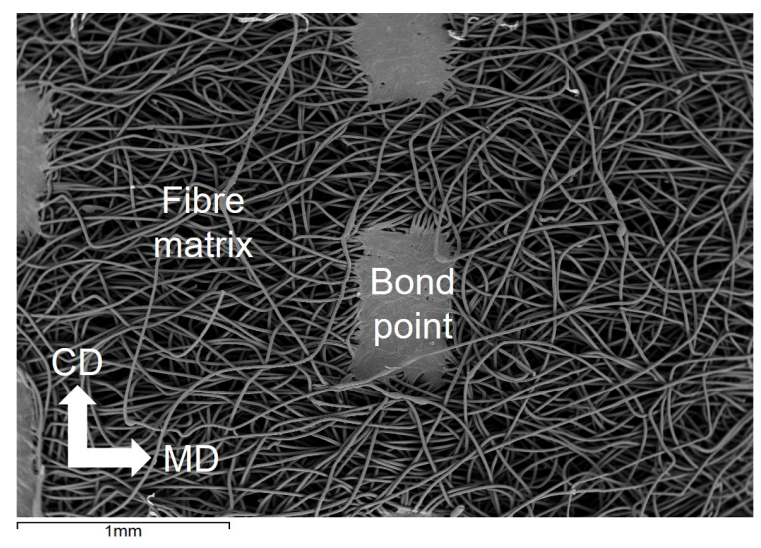




\subsubsection{Experimental Procedure}

In characterization of a fibrous network, fibre- and fabric-level experimental tests are required to understand its deformation and damage mechanisms. Fibres are fundamental components of random fibrous networks, playing key roles in deformation mechanisms at micro-level. They, therefore, should be tested with tensile tests to quantify their material properties. Subsequently, the material properties are implemented in FE models of the fibrous networks. In addition to the fibre tests, nonwoven fabrics were tested in tension to verify the FE models and to investigate their notch sensitivity. Evolution of deformation and damage in experimental and numerical investigations were compared.

\subsubsection{Single-fibre Tests}

As discussed, material properties of individual fibres should be incorporated into FE models of random fibrous networks. One way to obtain these properties is to test the fibres under tensile conditions. It is known that physical properties of fibres are sensitive to manufacturing parameters, such as calendaring pressure and temperature. This means that their mechanical properties after the web-bonding process are different than those before the process. The data from tensile tests on processed and unprocessed fibres demonstrated significant differences, especially in their strengths (Mueller and Kochmann, 2004). Failure stress and corresponding strain values of processed and unprocessed fibres for various strain rates are shown in Table 8.1.

Employing a realistic and pragmatic approach, individual fibres extracted from nonwoven fabrics were used in a universal testing system (Instron ${ }^{\circledR}$ Micro Tester 5848) to obtain strain-rate-dependent elastoplastic properties of fibres and to establish damage criteria for them in a FE model. Additionally, experimental observations pointed out that fibres of thermally point-bonded nonwovens failed at peripheries of these points. So, fibres were extracted from the nonwoven fabric with their bond points at the edges (see Fig. 8.5 (a)). This sample was placed between grips in the

Table 8.1 Failure stress and strain values of processed and unprocessed fibres at various strain rates (reproduced from Farukh et al., 2013)

\begin{tabular}{lccc}
\hline Strain rate (1/s) & Fibre type & Failure stress (MPa) Strain at failure stress \\
\hline 0.01 & processed & $481 \pm 48$ & $1.29 \pm 0.67$ \\
& unprocessed & $1299 \pm 95$ & $2.93 \pm 0.78$ \\
0.1 & processed & $315 \pm 28$ & $1.05 \pm 0.11$ \\
& unprocessed & $998 \pm 57$ & $1.77 \pm 0.09$ \\
0.5 & processed & $241 \pm 53$ & $0.79 \pm 0.10$ \\
& unprocessed & $818 \pm 60$ & $1.49 \pm 0.14$ \\
\hline
\end{tabular}

Page: 137 job: ABK_editor macro: svmult.cls date/time: 4-Sep-2019/22:46 

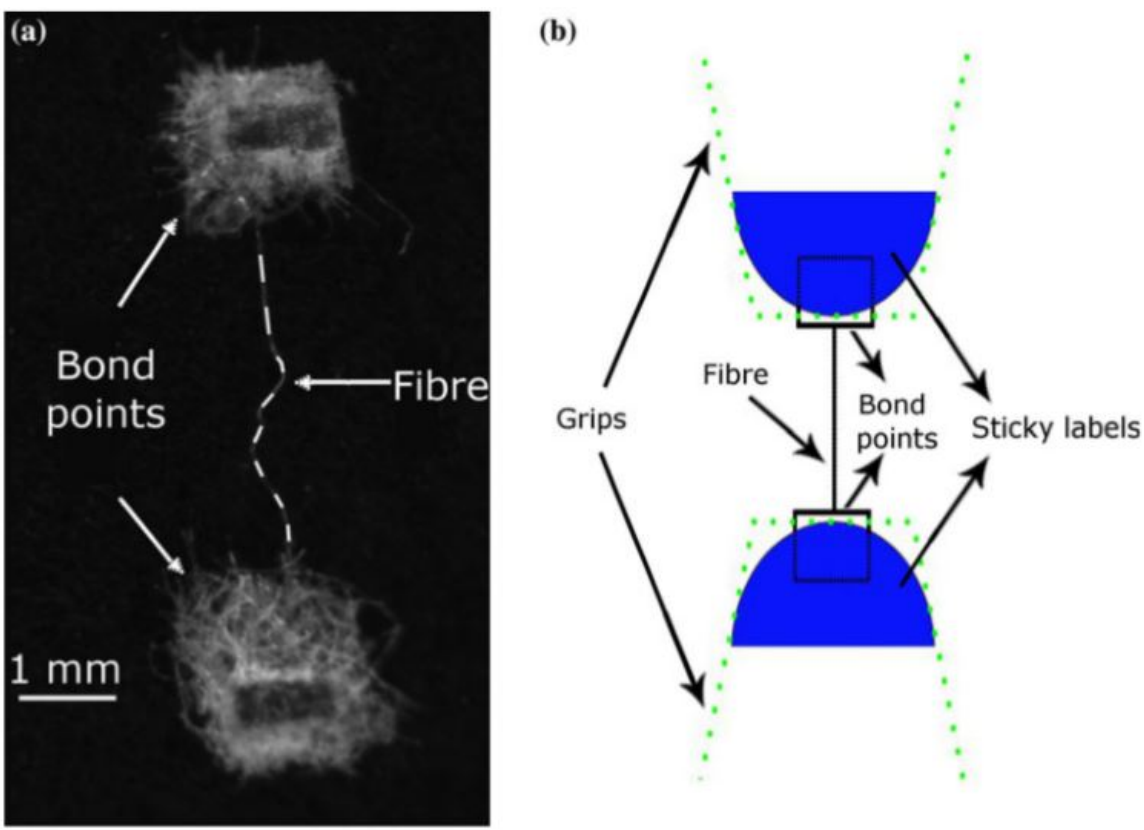

Fig. 8.5 (a) Single fibre with bond points at each end; (b) schematic of single-fibre specimen and its fixture Farukh et al. (2013)

tensile tester, shown in Fig. 8.5 (b). To prevent any slippage between the fibre and the grips, sticky labels were attached to the bond points at the fibre edges. A schematic of the single-fibre specimen in grips is presented in Fig. 8.5 (b). The specimen was tested in Instron ${ }^{\circledR}$ Micro Tester 5848 with a high-precision $5 \mathrm{~N}$ load cell. A detailed procedure of this test was explained in Farukh et al. (2013); Sabuncuoglu et al. (2013). The tests were performed with a displacement control of cross-head movement, enabling a constant strain rate. The tensile tests were performed on processed PP fibres with various levels of strain rates - 0.01, 0.1, $0.51 / \mathrm{s}$ - and mean true stress-strain responses were calculated. Using a linear elasticity theory, the contribution of elastic strain was obtained and, then, removed from the true stress-strain curves, resulting in true stress-plastic strain curves (Fig. 8.6). For repeatability of the results, eight fibres for each strain rate were used. While calculating the stress-strain curves, an assumption of perfectly circular fibre cross-section was made, and the initial fibre diameter was $18 \mu \mathrm{m}$, measured from SEM images and justified with the manufacturer data. The curves in Fig. 8.6 were used as input into the FE models of random fibrous networks. Nonwoven-fabric tests verified the reliability of the FE models. Single-fibre tests provided the elastic modulus and the Poisson's ratio of fibres as $350 \mathrm{MPa}$ and 0.42 Farukh et al. (2014b). It was assumed that the material properties of bond points were the same. 


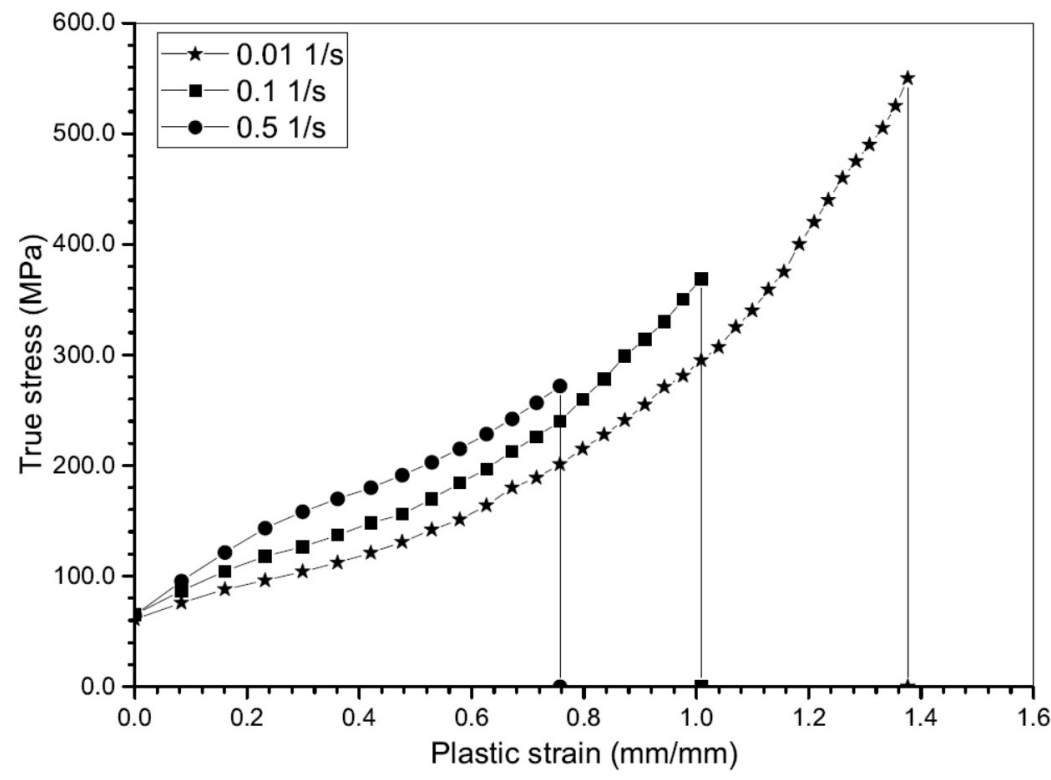

Fig. 8.6 Mean true stress-plastic strain curves for PP fibre at $0.01,0.1,0.51 / \mathrm{s}$ strain rates

\subsubsection{Fabric Tests}

Coupon specimens with square gauge area with the dimension of $25 \times 25 \mathrm{~mm}^{2}$ were prepared for fabric tests. For the notch-sensitivity analysis, four types of notches - circular, square, slit and diamond (see Fig. 8.7) - with the main dimension of $6.35 \mathrm{~mm}$ (D) were produced in the centres of the specimens using a surgery knife. To assess the effect of notches on deformation and damage behaviours of fibrous networks, non-damaged specimens were prepared and tested under tensile load. The specimens with notched and virgin geometries were tested in MD with a Hounsfield testing system (shown in Fig. 8.8) with a constant strain rate of $0.21 / \mathrm{s}$. In the

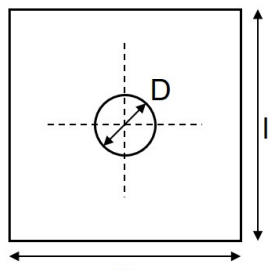

W

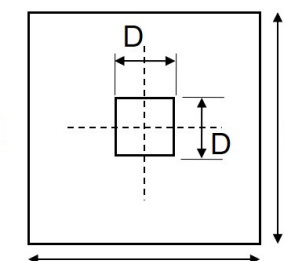

W

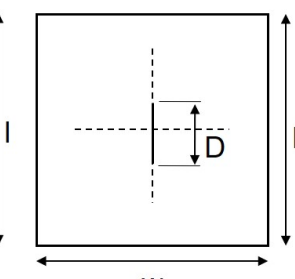

W

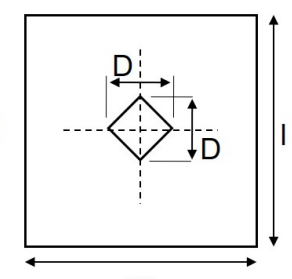

W

Fig. 8.7 Dimensions of gauge areas of coupon specimens with four different shapes of central notches: circular, square, slit and diamond 


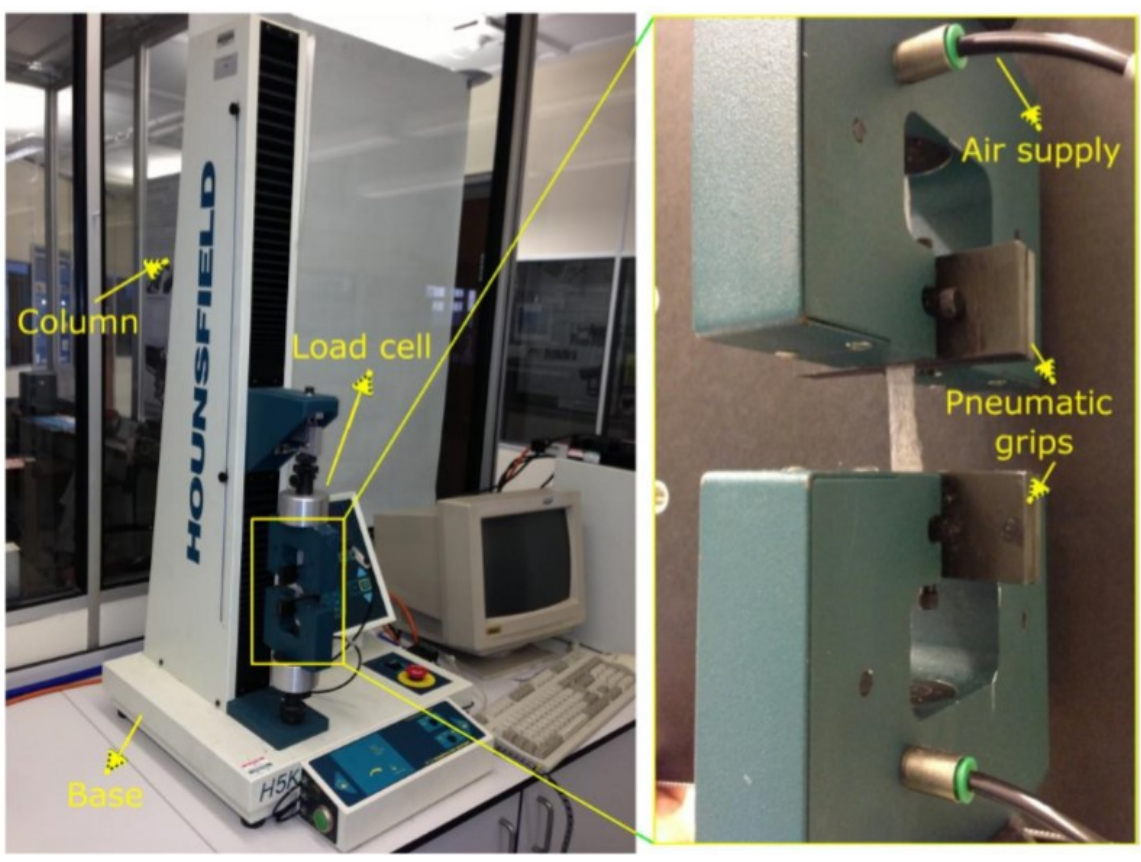

Fig. 8.8 Specimen in Hounsfield Benchtop Tester with pneumatic grips Farukh (2013)

experimental system, the specimens were fixed between pneumatic grips preventing any failure on clamped areas.

\subsection{Numerical Investigations}

For many years, researchers attempted to explain the mechanical behaviour of the fibrous networks using numerical models. In a pioneer work, H. L. Cox derived stiffness and strength of paper as a fibrous network in terms of orientation distribution of fibres and their elastic properties (Cox, 1952). M. A. Narter extended H. L. Cox's model for three-dimensional anisotropic fibrous networks (Narter et al., 1999). The finite-element method was one of the employed numerical methods; it is used in this research to simulate deformation and damage behaviours of random fibrous networks. FE models of such networks can be modelled with continuous and discontinuous approaches. Continuous models consider homogenisation of the networks to simulate macroscopic features of deformation; however, individual fibres are not generated and their failure caused by high strains can not be calculated in continuous models. Conversely, discontinuous models aim to elucidate the effect of microstructure of the material, incorporating specific features, such as randomly distributed fibres, bond 
points etc. into FE simulations. In this way, underpinning deformation and damage processes can be explained under variety of loading conditions.

\subsubsection{Discontinuous Finite-element Modelling of Random Fibrous Network}

A micromechanical model was developed to obtain an insight into detailed features of deformation and damage of a random fibrous network. First, geometric features of microstructure of an undeformed network, such as dimensions of bond points and their pattern, were characterized with SEM; these data are available in (Sozumert et al., 2018). Additionally, the orientation distribution function (ODF) of fibres was computed with a Hough-transform-based algorithm (Demirci et al., 2012). The algorithm of the code, generating the FE model of a fibrous network, is illustrated in Fig. 8.9. Using this code, FE models of the coupon specimens with and without notches were generated in a commercial FE software, MSC Marc-Mentat. The notches were placed in the centre of specimen using $x-y$ coordinate system and the same bond point pattern implemented for all notch shapes. The resultant FE model of the fibrous network is presented in Fig. 8.10 (a) with applied boundary conditions. In the FE software, fibres and bond points were modelled with two-node truss elements and thin-shell elements, respectively. Then, in a weak formulation for a momentum equation for a truss-shell system, the total internal virtual work $\delta W_{\text {int }}$, which is a sum of contributions from truss and shell elements, is equal to the total external virtual work $\delta W_{\text {ext }}$ :

$$
\begin{array}{r}
\delta W_{\mathrm{int}}=\delta W_{\mathrm{ext}}, \\
\delta W_{\mathrm{int}}=\sum_{i=1}^{n} \delta W_{\mathrm{int}}^{\mathrm{truss}}+\sum_{i=1}^{m} \delta W_{\mathrm{int}}^{\text {shell }} .
\end{array}
$$

The internal virtual work for a truss - in absence of transverse shear and moments - and for a shell element based on a Discrete Kirchhoff Theory in absence of shear deformation in $x$ and $y$ (in-plane) are given by

$$
\begin{array}{r}
\delta W_{\mathrm{int}}^{\mathrm{truss}}=\int_{0}^{L}\left(N_{z} \delta \varepsilon_{z}\right) d z, \\
\delta W_{\mathrm{int}}^{\text {shell }}=\int_{0}^{L}\left(M_{x} \delta \kappa_{z}+2 M_{x y} \delta \kappa_{x y}+M_{y} \delta \kappa_{y}\right) d A .
\end{array}
$$

The virtual work of a truss is defined by the work done by axial force $N_{z}$, where, in a local coordinate system of a truss, $z$-axis coincides with the longitudinal axis. Additionally, the virtual work of a shell is the sum of a virtual work done by the bending moments $M_{x}$ and $M_{y}$, and the twisting moment $M_{x y}$, where $\kappa_{y}, \kappa_{z}$ and

Page: 141 job:ABK_editor macro:svmult.cls date/time: 4-Sep-2019/22:46 


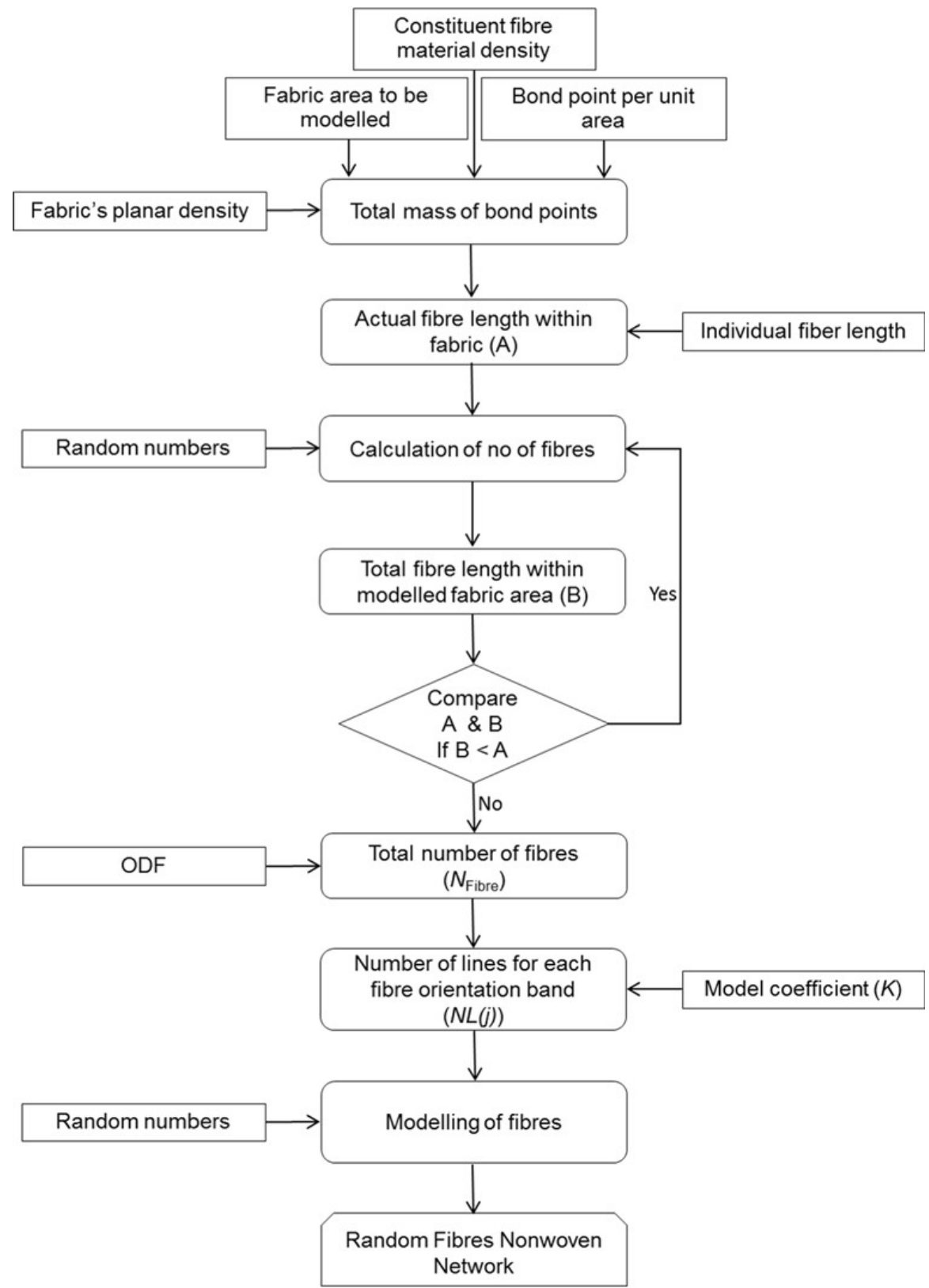

Fig. 8.9 Algorithm to generate a random fibrous network Farukh et al. (2014a) 
(a)

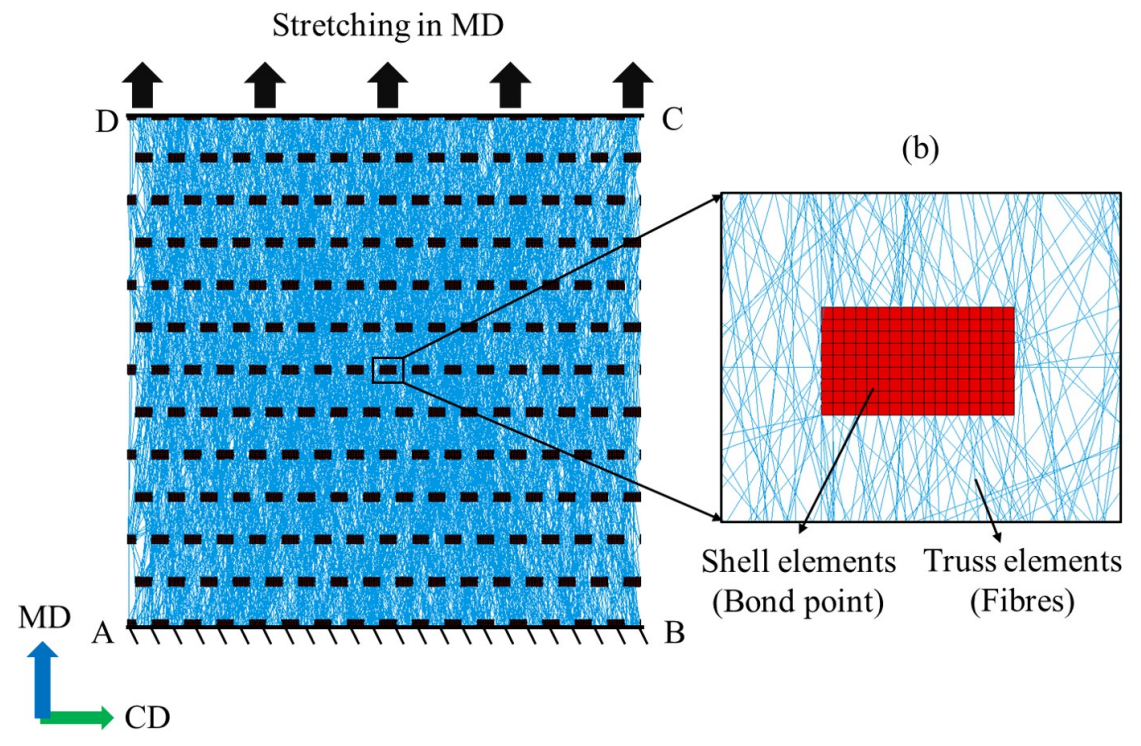

Fig. 8.10 (a) FE model with boundary conditions; (b) zoomed-in view of bond point and fibres

$\kappa_{x y}$ are curvatures. In the FE model, the bond points were discretised by thin-shell elements due to their relatively low thickness compared to their length and width (Fig. 8.4). This analysis considered that the bond points and fibres were represented one-to-one in the discrete FE models. Together with this, material behaviour of single fibres was incorporated in the incremental form of elastic-plastic stress-strain relations (MSC-Software, 2016; Belytschko et al., 2013). further information about the constitutive relations can be found in Sozumert et al. (2018).

Some fundamental assumptions of the research should be mentioned: fibre curvature and interactions were neglected. For tensile loading, the curvature might however increase the toughness of the material by controlling the maximum stress and strain.

The simulations of tensile tests were performed with the FE models with virgin and notched configurations by applying a set of boundary conditions to the lower boundary $\mathrm{AB}$ and the upper boundary $\mathrm{CD}$ (see Fig. 8.10 (a)):

(i) the nodes on $\mathrm{AB}$ were fully fixed, reflecting the exact boundary conditions of pneumatic grips in the experimental tests;

(ii) the nodes on $\mathrm{CD}$ were moved with a uniform axial displacement in MD.

It is noted that FE simulations were carried out with an implicit solver under quasistatic loading conditions by using a large-deformation formulation. 


\subsection{Results and Discussion}

\subsubsection{Experimental Observations}

To investigate the effect of notch shape on deformation and damage behaviour, the nonwoven specimens with a virgin microstructure and various notch geometries were tested in machine direction under tensile loading and force-displacement readings were obtained from the Instron ${ }^{\circledR}$ system. To make a comparison between the virgin and notched cases, the respective force curves were normalised by using the effective width (the difference between the specimen's width and the notch width). The (mean) normalised force-extension curves of these specimens in MD are shown in Fig. 8.11 (a). Five specimens were tested for each geometric configuration and a significant scatter in force-displacement data after the onset of damage was observed; therefore,

(a)

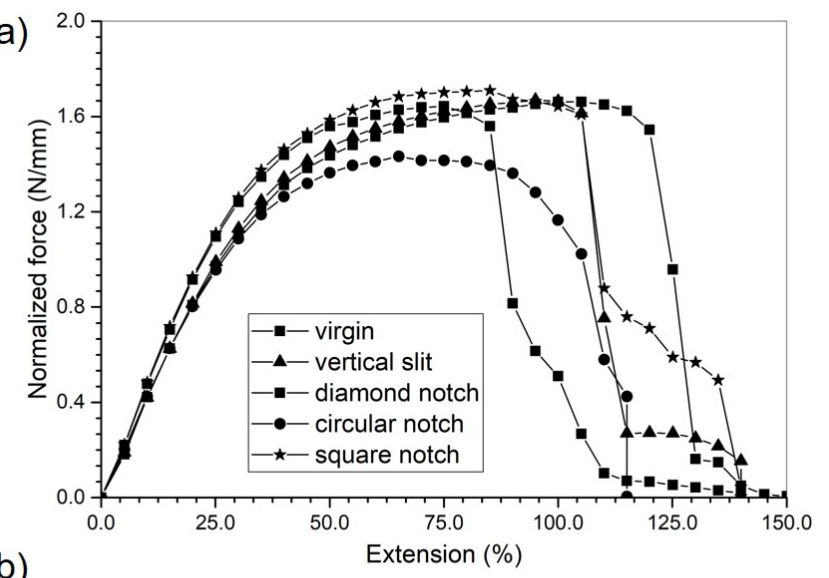

(b)
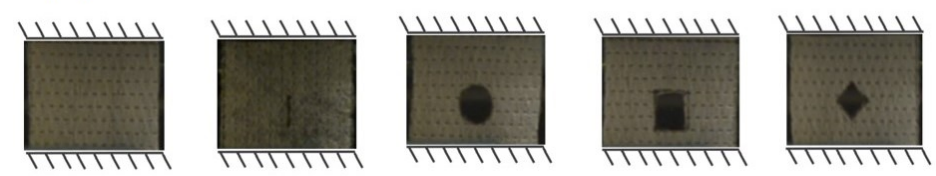

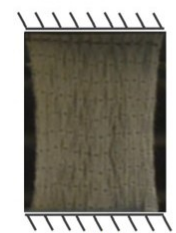

(1)

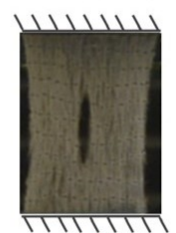

(2)

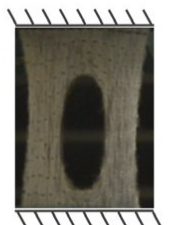

(3)

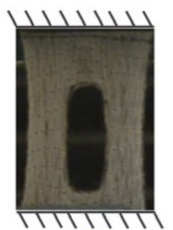

(4)

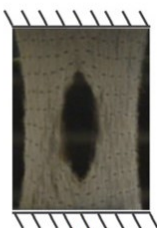

(5)

Fig. 8.11 (a) Experimental mean force-extension curves for virgin and notched specimens tested in MD. (b) Damage evolution in various nonwoven specimens under 60\% extension in MD: (1) virgin; (2) vertical slit; (3) circular notch; (4) square notch; (5) diamond notch (Sozumert et al., 2018) 
mean curves were plotted. Based on Fig. 8.11 (a), deformation behaviour of the fibrous network was sensitive to the notch shape, but to different extent. Mean tensile strength of specimens, however, was insensitive to the vertical slit. The underlying reason for this is the fact that the ODF of the selected fibrous network showed a preferential orientation of fibres in MD as compared to $\mathrm{CD}$. As the vertical slit notch was opened, the amount of fibres cut, making contribution to the load-carrying capacity, was negligible.

Damage evolution of the nonwoven specimens with virgin and damaged configurations is shown in Fig. 8.11 (b). Several deformation processes related to constituting fibres of the random fibrous network take place in microstructure:

(i) fibre straightening from initial curved form;

(ii) realigning of fibres towards the loading direction;

(iii) fibre stretch and failure.

From the perspective of macrostructure, at the early stage of tensile stretch, the central notches grew in both MD and CD. After the realignment of fibres was completed, the speed of damage growth in MD was much faster than the one in CD.

Microstructural observations of virgin specimens of thermally-point bonded nonwovens under tensile load revealed that, after straightening of fibres, the fibres were rotated towards the loading direction and their progressive failure followed this process when their failure strain was reached (Farukh et al., 2014b). Experiments showed that these deformation and damage mechanisms were independent of the basis weight, and the strain rate of the tensile test did not change the sequence of deformation and damage phenomena (Ridruejo et al., 2011).

The changes in the microstructure of the virgin nonwoven sample at the different level of extensions are showed in Fig. 8.12. Fibres were bundled around bond points and weak areas at arbitrary locations, where lower density of fibres was observed. These weak areas, i.e. zones of damage localisations, expanded with further deformation, with fibres failing in the areas supporting this expansion (Sozumert et al., 2018). Arrows in Fig. 8.12 indicate fibres failed as a result of large strain. For the specimens with square, diamond and circular notches, the position of damagelocalisation areas was more predictable as compared to virgin specimens: they were near the notches. Re-orientation of fibres towards the loading direction toughened the material, while sharp edges of the notches were blunted. Bond points in various locations of the fibrous network were rotated by different amounts as a result of a non-uniform distribution of individual fibres in the network. Deformation and load transfer mechanisms between bond points and their attached fibres are highly unpredictable since the load was transferred via random sets of links between bond points and fibres (i.e. discontinuous load-transfer mechanism). Therefore, local loads were diffused rather than concentrated with stress singularity near a notch as in continuous materials (such as metals).

The numerical results (Fig. 8.11 (a)) provided an evidence that the levels of strength of specimens with virgin and notches were almost the same. Apparently, the specimens with circular holes showed poorer tensile performance than the rest of the specimens. A limited investigation on circular- and longitudinal-notched specimens

Page: 145 job:ABK_editor macro: svmult.cls date/time: 4-Sep-2019/22:46 


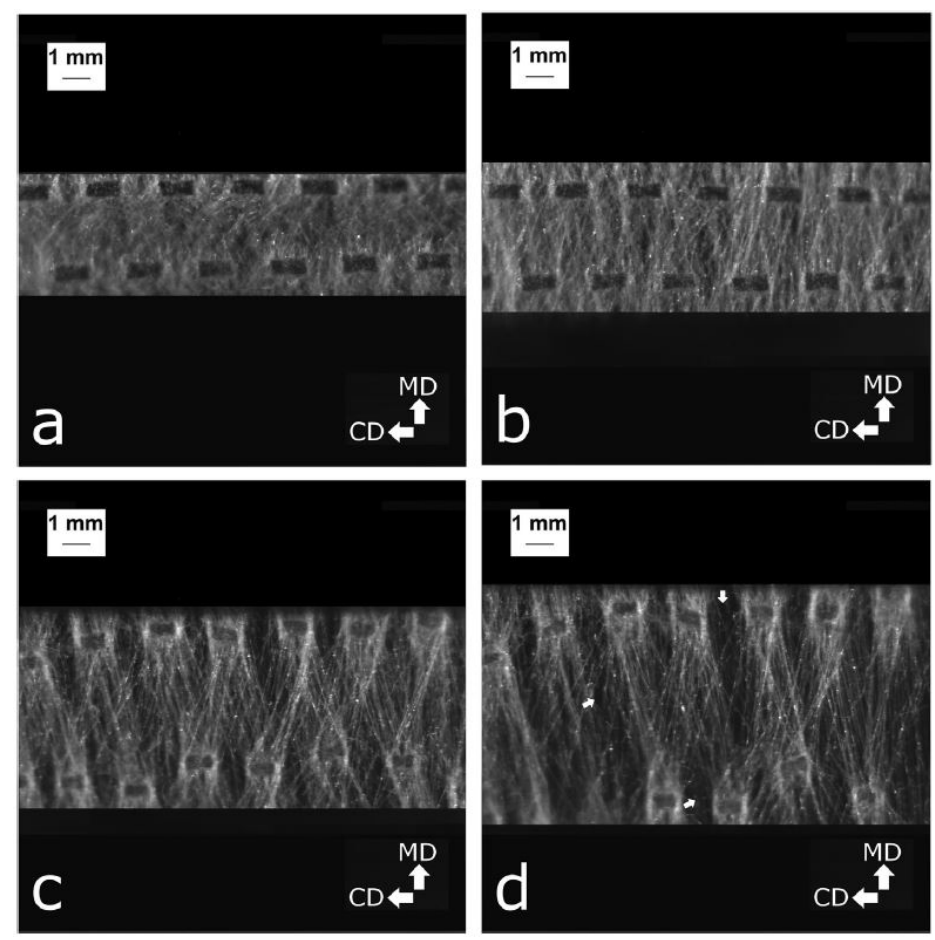

Fig. 8.12 Deformation and damage mechanisms of virgin nonwoven specimen: (a) $0 \%$ extension; (b) $25 \%$ extension; (c) 50\% extension; (d) $80 \%$ extension (Farukh et al., 2014b)

was conducted in Rawal et al. (2013), revealing the similar detrimental effect of circular notches on the tensile response of needle-punched hybrid nonwovens. One hypothesis is that a higher number of fibres contributing to the load-carrying capacity was affected as compared to other notch cases.

\subsubsection{Finite Element Simulations}

In the FE simulations, the true stress-true strain curve $(0.011 / \mathrm{s}$ in Fig. 8.6) was implemented into an elastic-plastic material model with von Mises plasticity in FE software. This curve showed a softer mechanical response than that used in simulations in Sozumert et al. (2018). Throughout this investigation, the strain rate effects on deformation and damage behaviour were not considered. A maximum-strain damage criterion for fibres was implemented into the FE models of the nonwoven specimens that were stretched until they failed completely. The damage parameter $D$ and the failure criteria are then expressed as 


$$
\text { if } D=\left(\frac{\varepsilon_{z}}{\varepsilon_{\mathrm{f}}}\right) \geq 1.0 \text {, fibre fails. }
$$

where $\varepsilon_{z}$ and $\varepsilon_{\mathrm{f}}$ are the longitudinal logarithmic strain (simulation) and the mean $\log$ arithmic failure strain (experiment), respectively. In simulations, mono-component fibres were assumed to show a fully isotropic material behaviour, and the failure stress $\varepsilon_{\mathrm{f}}=1.4$ was taken from single-fibre tests. In the FE models, each truss elements represented a fibre segment and, as the longitudinal strain $\varepsilon_{z}$ in it exceeded the failure stress $\varepsilon_{\mathrm{f}}$, it was assumed that the fibre (or truss element) failed and it was deleted.

Tensile behaviour of nonwoven specimens with virgin, vertical-slit, square and circular notches was successfully simulated with the developed model and the assigned damage criterion for fibres. Microstructural changes at different levels of extensions were analysed. Strains of fibre segments were obtained from the deformed FE models of virgin nonwoven specimens and normalised by the failure strain of fibres. A histogram of the normalised fibre strains at $40 \%, 60 \%, 80 \%$, and $100 \%$ fabric extensions is presented in Fig. 8.13. More than $90 \%$ of fibre segments shows normalised strains in the range between 0-0.4 in 40\% extension, far below the amount required for failure. As the FE model was stretched further, almost $10 \%$ of fibre segments approached to the value of 1.0. A further increase in the extension value resulted in failure of constituent fibres in the virgin specimen, leading to a change of the network's microstructure. It was therefore observed that some of fibres in the loading direction (MD) shifted back to their original position due to local stress

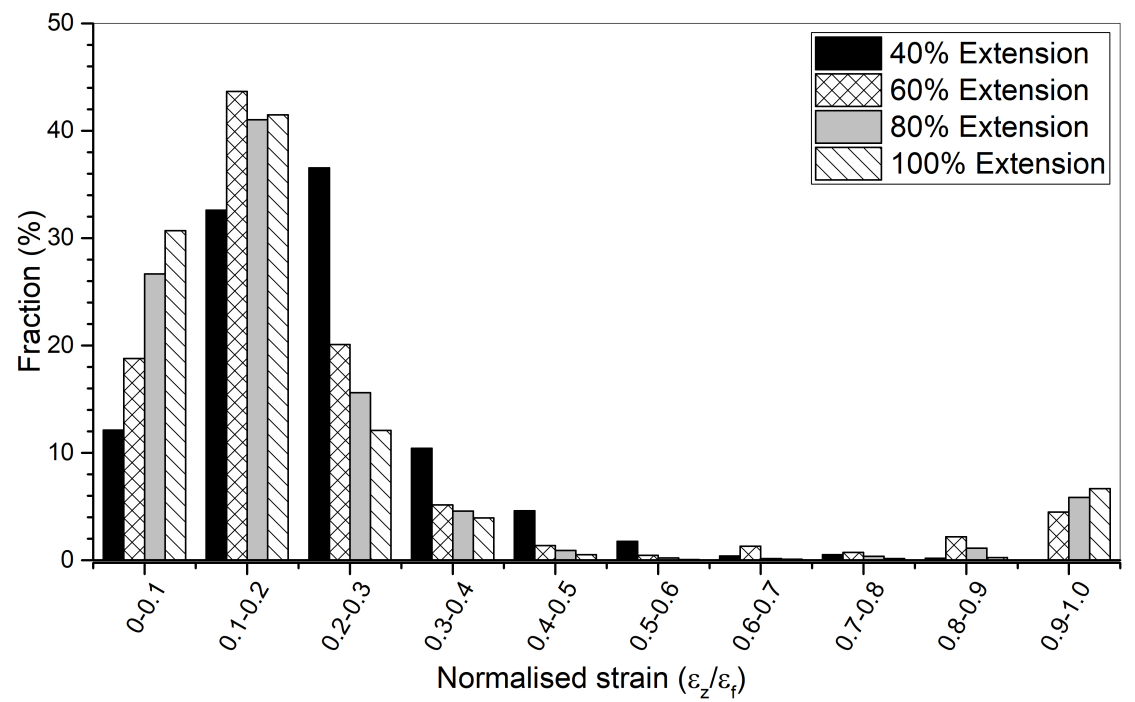

Fig. 8.13 Distributions of fibre strains (normalised by failure strain of fibres) in virgin specimen (simulation) for fabric extension of $40 \%, 60 \%, 80 \%$, and $100 \%$ 
release. However, as the network was further stretched, those fibres were re-aligned in MD.

Images of damage patterns for the virgin, slit-, circular- and square-notched specimens for $0 \%$ and $60 \%$ extensions were obtained from experimental and numerical tensile tests (Fig. 8.14). The FE models with various geometries were capable to

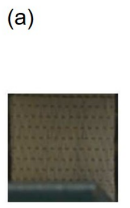

$0 \%$ extension

(b)

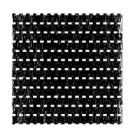

$0 \%$ extension

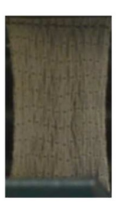

$60 \%$ extensio

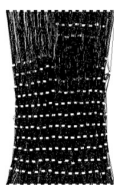

$60 \%$ extension

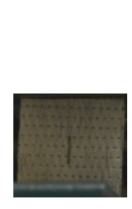

$0 \%$ extension $60 \%$ extension

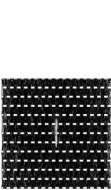

$0 \%$ extension
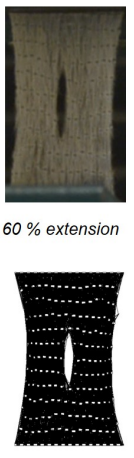

$60 \%$ extension

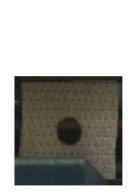

$0 \%$ extension

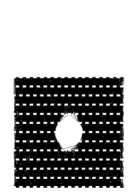

$0 \%$ extension $60 \%$ extension

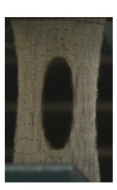

$60 \%$ extension

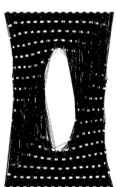

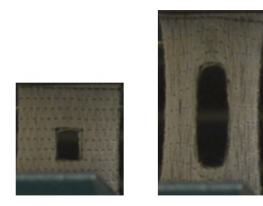

$0 \%$ extension $\quad 60 \%$ extension

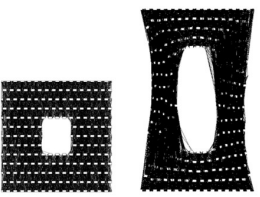

$0 \%$ extension $60 \%$ extension

Fig. 8.14 Comparison of experimental (a) and simulated damage (b) patterns for virgin, slit-notch, circular-notch and square-notch specimens for $0 \%$ and $60 \%$ extensions

demonstrate the main deformation and damage features, such as damage growth in orthogonal directions and necking behaviour. Fibres in the fibrous network were randomly oriented at $0 \%$ extension. As they were subjected to uniaxial stretch, the notches in the specimens opened and fibres were re-aligned towards the loading direction (MD). The amount of re-alignment was not equally distributed throughout the notched specimens; therefore, failure of individual fibres were more localised in certain regions where the fibres were more preferentially aligned. The FE simulations demonstrated that fibres bundles were formed in front of notch tips, accommodating mechanical strain and resisting crack propagation, thus toughening the fabric (Koh et al., 2013).

In the experiments, sharp edges in the notched specimens were blunted, and this geometric variation due to fabric deformation was well captured in the FE simulations. The square and slit notches became a rectangle-like damage area and an elliptical hole, respectively. Like the slit notch, the circular hole was transformed into an elliptical hole, but with a relatively lower aspect ratio. As the specimens in the simulations were stretched, re-alignments of fibres towards the loading direction was followed by re-alignments of bond points. Their distribution in the virgin specimen was quite similar to that in the slit-notch specimen at $30 \%$ extension, where the aspect ratio of ellipse in the slit notch increased. Alignments of bond points around the square and circular notches were almost the same.

In the FE models, fibres along selected paths were tracked to assess the localisation of strain; Fig. 8.15 shows these paths for virgin, slit-notch, square-notch and circularnotch specimens. They were chosen in the centre of specimens, parallel to the loading 


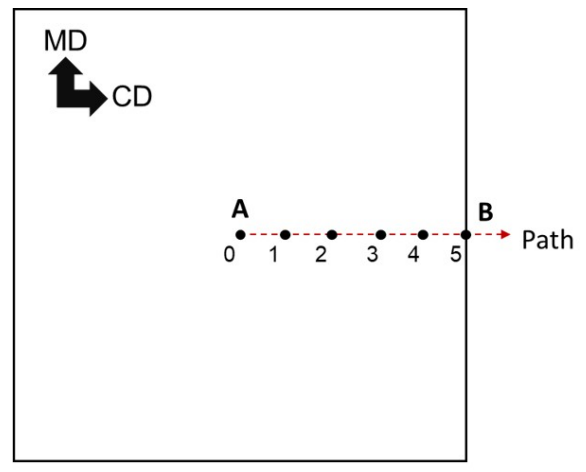

(a)

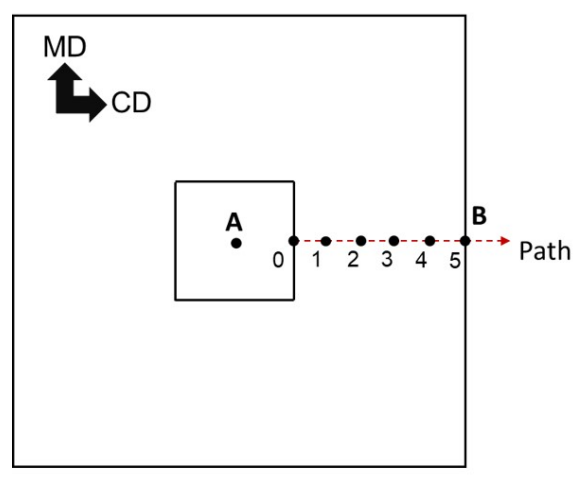

(c)

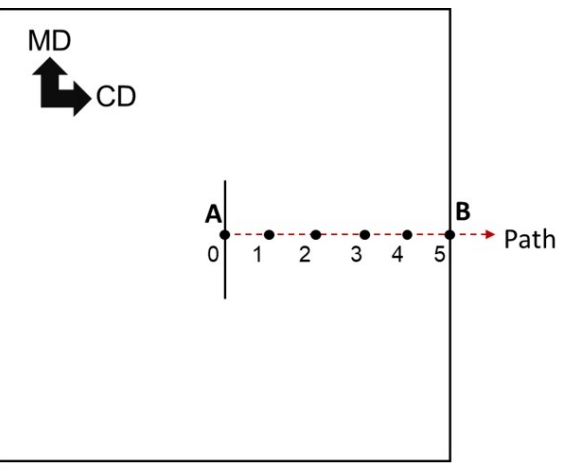

(b)

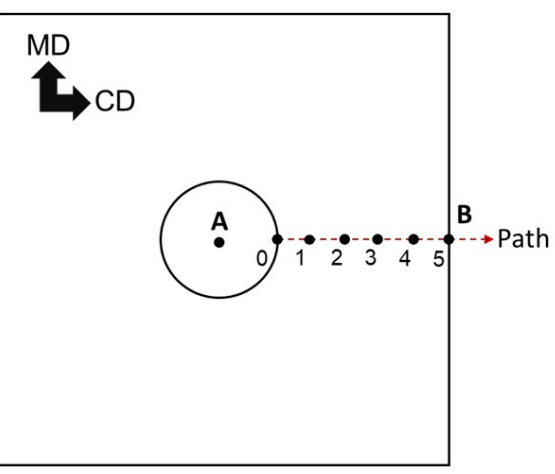

(d)

Fig. 8.15 Paths selected for specimens with virgin (a), slit-notch (b), square-notch (c) and circular-notch (d) geometries

direction and divided into five equal intervals. Logarithmic axial strains $\varepsilon_{z}$ of fibres in these intervals were calculated for 60\% (Fig. 16 (a)) and 80\% (Fig. 16 (b)) stretching of the networks and averaged for each interval.

Non-uniform strain distributions were obtained for the tracked paths. Apparently, the normalised strains increased towards the notch tips (node-0) in Fig. 8.16's (a) and (b), demonstrating notch-sensitivity. Additionally, the highest normalised (averaged) strains were observed at the tip of slit notch. Due to lateral contraction and fibre alignment, the virgin specimen showed a nonlinear behaviour along the path, but lower strains as compared to the notched specimens. In terms of location (with the same x-y coordinates, red-circled in Fig. 8.16's (a) and (b)), all cases of notches demonstrated similar damage performance. The slit-notched specimen, however, resulted in a sharper increase in the vicinity of the notch tip than the other notch cases, i.e. more localised strain around it. Unlike the slit-notched specimen, the square-notch one dispersed strain more out of the notch region. 
(a)

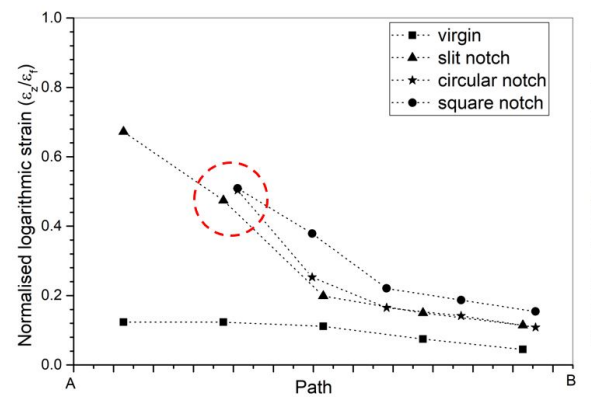

(b)

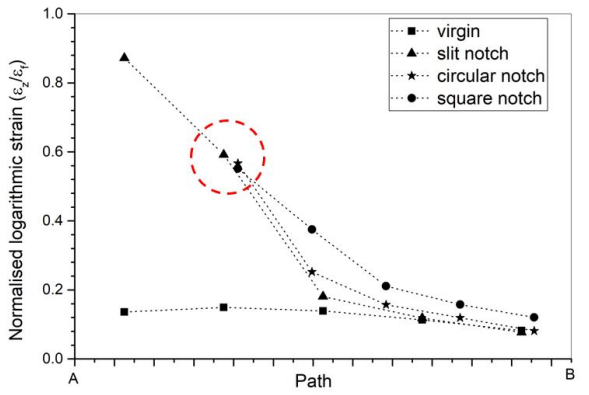

Fig. 8.16 FE-based calculated distributions of fibre strains for specimens with virgin, slit-notch, square-notch and circular-notch geometries for various levels of network stretching: (a) $60 \%$; (b) $80 \%$ extensions

With application of digital image processing of calculated damage patterns of the notched specimens, changes in damage length $\Delta \mathrm{L}(\mathrm{MD})$ and width $\Delta \mathrm{W}$ (CD) for various levels of fabric stretching were measured and normalised by initial length $\mathrm{L}_{0}$ and width $\mathrm{W}_{0}$, respectively. The damage growth in MD and in CD are demonstrated in Fig. 8.17. For all the notch cases, the damage growth in MD was almost linearly proportional to fabric strain. For damage growth in MD for the square- and circularnotched specimens showed similar behaviour and higher than that of the slit-notch specimen. As for the growth in CD, square- and circular-notched specimens were tracked and a nonlinear response was observed (Fig. 8.17 (b)). Opposite to the behaviour in $\mathrm{MD}$, the square notch grew larger in $\mathrm{CD}$ than the circular notch. It increased at the initial stage, till 0.4 of fabric strain and started shrinking. Similarly, the circular notch shrank after a certain extent of stretching, followed by a decrease, even somehow below the initial notch width $\mathrm{W}_{0}$.

\subsection{Summary and Conclusions}

This study explained experimental and numerical methods for characterizing deformation and damage behaviour of random fibrous networks, with a special focus on deformation and damage evolution from local (fibre) to global (fabric) levels in a nonwoven material. Tensile tests were conducted on polymer fibres extracted from the nonwoven in order to obtain their material properties. The fibres demonstrated a nonlinear time-dependent material response to stretching. Undamaged nonwoven samples and ones with various notches were experimentally and numerically analysed. This was accompanied by development of discrete FE models imitating a real material microstructure. The FE models reproduced the deformation and damage mechanisms of fibrous networks at micro- and macro-scales. It was demonstrated that the notch shape had a significant effect on toughness of the network, with the 
circular notch having the most detrimental effect as compared to slit, square and diamond notches. In the FE simulations, using image processing, damage growth was tracked in two main orthogonal directions (MD and $\mathrm{CD}$ ) as the specimens were stretched in MD. It was found that the rate of damage growth differed in various directions. This behaviour is linked to the main deformation and damage mechanisms of the fibrous network: rearrangement, stretching and failures of fibres.

\section{References}

Belytschko T, Liu WK, Moran B, Elkhodary K (2013) onlinear Finite Elements for Continua and Structures. Wiley

Buerzle W, Mazza E (2013) On the deformation behavior of human amnion. Journal of Biomechanics 46(11):1777 - 1783, DOI 10.1016/j.jbiomech.2013.05.018

Cheng Q, Wang S (2008) A method for testing the elastic modulus of single cellulose fibrils via atomic force microscopy. Composites Part A: Applied Science and Manufacturing 39(12):1838 - 1843, DOI 10.1016/j.compositesa.2008.09.007

Cox HL (1952) The elasticity and strength of paper and other fibrous materials. British Journal of Applied Physics 3(3):72-79, DOI 10.1088/0508-3443/3/3/302

Demirci E, Acar M, Pourdeyhimi B, Silberschmidt VV (2012) Computation of mechanical anisotropy in thermally bonded bicomponent fibre nonwovens. Computational Materials Science 52(1): 157 - 163, DOI 10.1016/j.commatsci.2011.01.033, proceedings of the 20th International Workshop on Computational Mechanics of Materials - IWCMM 20

Farukh F (2013) Experimental and numerical analysis of deformation and damage in thermally bonded nonwoven material. PhD thesis, Loughborough University

Farukh F, Demirci E, Acar M, Pourdeyhimi B, Silberschmidt VV (2012) Strength of fibres in low-density thermally bonded nonwovens: An experimental investigation. Journal of Physics: Conference Series 382:012018, DOI 10.1088/1742-6596/382/1/012018

(a)

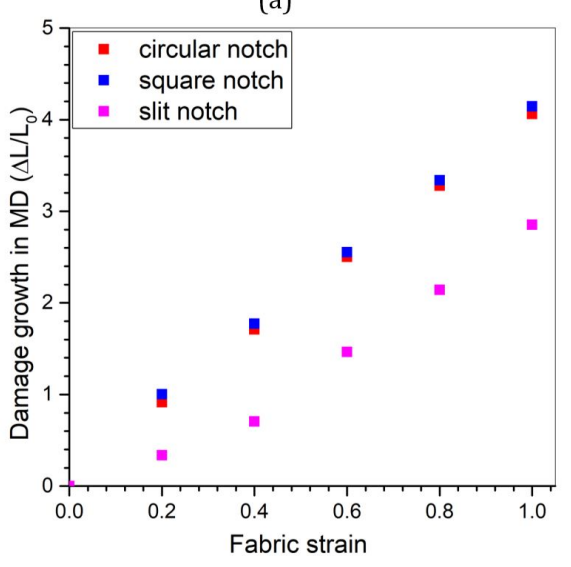

(b)

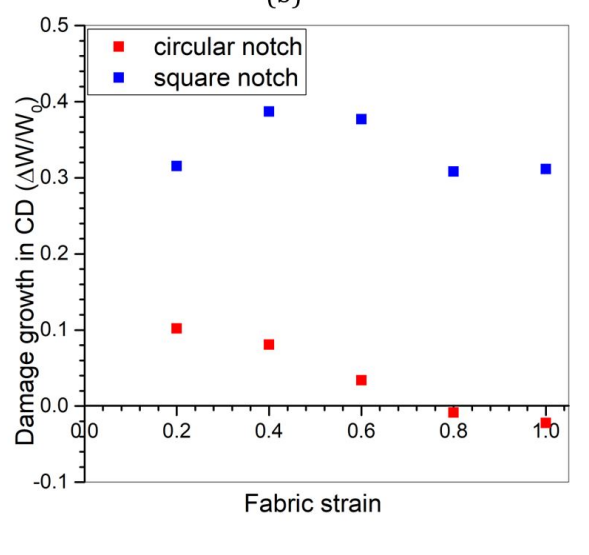

Fig. 8.17 Damage growth in MD (a) and CD (b) 
Farukh F, Demirci E, Acar M, Pourdeyhimi B, Silberschmidt VV (2013) Meso-scale deformation and damage in thermally bonded nonwovens. Journal of Materials Science 48(6):2334-2345, DOI 10.1007/s10853-012-7013-y

Farukh F, Demirci E, Acar M, Pourdeyhimi B, Silberschmidt VV (2014a) Large deformation of thermally bonded random fibrous networks: microstructural changes and damage. Journal of Materials Science 49(11):4081-4092, DOI 10.1007/s10853-014-8100-z

Farukh F, Demirci E, Sabuncuoglu B, Acar M, Pourdeyhimi B, Silberschmidt VV (2014b) Numerical analysis of progressive damage in nonwoven fibrous networks under tension. International Journal of Solids and Structures 51(9):1670 - 1685, DOI 10.1016/j.ijsolstr.2014.01.015

Gao X, Shi Z, Kusśmierczyk P, Liu C, Yang G, Sevostianov I, Silberschmidt VV (2016a) Timedependent rheological behaviour of bacterial cellulose hydrogel. Materials Science and Engineering: C 58:153 - 159, DOI 10.1016/j.msec.2015.08.019

Gao X, Shi Z, Liu C, Yang G, Silberschmidt VV (2016b) Fracture behaviour of bacterial cellulose hydrogel: Microstructural effect. Procedia Structural Integrity 2:1237 - 1243, DOI 10.1016/j. prostr.2016.06.158, 21st European Conference on Fracture, ECF21, 20-24 June 2016, Catania, Italy

Gao X, Sozumert E, Shi Z, Yang G, Silberschmidt VV (2017) Assessing stiffness of nanofibres in bacterial cellulose hydrogels: Numerical-experimental framework. Materials Science and Engineering: C 77:9 - 18, DOI 10.1016/j.msec.2017.03.231

Ghassemieh E, Acar M, Versteeg H (2002) Microstructural analysis of non-woven fabrics using scanning electron microscopy and image processing. Part 1: Development and verification of the methods. Proceedings of the Institution of Mechanical Engineers, Part L: Journal of Materials: Design and Applications 216(3):199-207, DOI 10.1177/146442070221600305

Hearle JWS, Stevenson PJ (1963) Nonwoven fabric studies: Part III: The anisotropy of nonwoven fabrics. Textile Research Journal 33(11):877-888, DOI 10.1177/004051756303301101

Isaksson P, Hägglund R (2009) Strain energy distribution in a crack-tip region in random fiber networks. International Journal of Fracture 156:1-9, DOI 10.1007/s10704-009-9340-9

Jubera R, Ridruejo A, González C, LLorca J (2014) Mechanical behavior and deformation micromechanisms of polypropylene nonwoven fabrics as a function of temperature and strain rate. Mechanics of Materials 74:14 - 25, DOI 10.1016/j.mechmat.2014.03.007

Kim HS, Pourdeyhimi B (2001) Computational modeling of mechanical performance in thermally point bonded nonwovens. Journal of Textile and Apparel, Technology and Management 1(4):1-7

Koh C, Strange D, Tonsomboon K, Oyen M (2013) Failure mechanisms in fibrous scaffolds. Acta Biomaterialia 9(7):7326 - 7334, DOI 10.1016/j.actbio.2013.02.046

Kulachenko A, Uesaka T (2012) Direct simulations of fiber network deformation and failure. Mechanics of Materials 51:1 - 14, DOI 10.1016/j.mechmat.2012.03.010

Kumar V, Rawal A (2017) Elastic moduli of electrospun mats: Importance of fiber curvature and specimen dimensions. Journal of the Mechanical Behavior of Biomedical Materials 72:6 - 13, DOI 10.1016/j.jmbbm.2017.04.013

Michielsen S, Pourdeyhimi B, Desai P (2006) Review of thermally point-bonded nonwovens: Materials, processes, and properties. Journal of Applied Polymer Science 99(5):2489-2496, DOI 10.1002/app.22858

MSC-Software (2016) Theory and user information

Mueller DH, Kochmann M (2004) Numerical modeling of thermobonded nonwovens. International Nonwovens Journal 13(1):56-62, DOI 10.1177/1558925004os-1300114

Narter MA, Batra SK, Buchanan DR (1999) Micromechanics of three-dimensional fibrewebs: constitutive equations. Proceedings of the Royal Society of London Series A: Mathematical, Physical and Engineering Sciences 455(1989):3543-3563, DOI 10.1098/rspa.1999.0465

Norouzi M, Boroujeni SM, Omidvarkordshouli N, Soleimani M (2015) Advances in skin regeneration: Application of electrospun scaffolds. Advanced Healthcare Materials 4(8):1114-1133, DOI 10.1002/adhm.201500001

Ovaska M, Bertalan Z, Miksic A, Sugni M, Benedetto CD, Ferrario C, Leggio L, Guidetti L, Alava MJ, Porta CAML, Zapperi S (2017) Deformation and fracture of echinoderm collagen

Page: 152 job:ABK_editor macro: svmult.cls date/time: 4-Sep-2019/22:46 
networks. Journal of the Mechanical Behavior of Biomedical Materials 65:42 - 52, DOI 10.1016/j.jmbbm.2016.07.035

Rawal A (2006) A modified micromechanical model for the prediction of tensile behavior of nonwoven structures. Journal of Industrial Textiles 36(2):133-149, DOI 10.1177/1528083706067691

Rawal A, Patel SK, Kumar V, Saraswat H, Sayeed MA (2013) Damage analysis and notch sensitivity of hybrid needlepunched nonwoven materials. Textile Research Journal 83(11):1103-1112, DOI $10.1177 / 0040517512467063$

Ridruejo A, González C, LLorca J (2010) Damage micromechanisms and notch sensitivity of glass-fiber non-woven felts: An experimental and numerical study. Journal of the Mechanics and Physics of Solids 58(10):1628 - 1645, DOI 10.1016/j.jmps.2010.07.005

Ridruejo A, González C, LLorca J (2011) Micromechanisms of deformation and fracture of polypropylene nonwoven fabrics. International Journal of Solids and Structures 48(1):153 162, DOI 10.1016/j.ijsolstr.2010.09.013

Ridruejo A, Jubera R, González C, LLorca J (2015) Inverse notch sensitivity: Cracks can make nonwoven fabrics stronger. Journal of the Mechanics and Physics of Solids 77:61 - 69, DOI 10.1016/j.jmps.2015.01.004

Sabuncuoglu B, Acar M, Silberschmidt VV (2012) Finite element modelling of thermally bonded nonwovens: Effect of manufacturing parameters on tensile stiffness. Computational Materials Science 64:192 - 197, DOI 10.1016/j.commatsci.2012.02.043, proceedings of the 21st International Workshop on Computational Mechanics of Materials (IWCMM 21)

Sabuncuoglu B, Demirci E, Acar M, Silberschmidt VV (2013) Analysis of rate-dependent tensile properties of polypropylene fibres used in thermally bonded nonwovens. The Journal of The Textile Institute 104(9):965-971, DOI 10.1080/00405000.2013.766391

Shiffler DA (1995) An examination of the stress-strain curve of crimped polyethylene terephthalate staple fibers. The Journal of The Textile Institute 86(1):1-9, DOI 10.1080/00405009508631305

Sozumert E, Farukh F, Sabuncuoglu B, Demirci E, Acar M, Pourdeyhimi B, Silberschmidt VV (2018) Deformation and damage of random fibrous networks. International Journal of Solids and Structures DOI 10.1016/j.jjsolstr.2018.12.012

Tan EPS, Goh CN, Sow CH, Lim CT (2005) Tensile test of a single nanofiber using an atomic force microscope tip. Applied Physics Letters 86(7):073115, DOI 10.1063/1.1862337

Yang W, Sherman VR, Gludovatz B, Schaible E, Stewart P, Ritchie R, Meyers MA (2015) On the tear resistance of skin. Nature Communications 6(6649), DOI 10.1038/ncomms7649 
